\section{The Diagnostic, Prognostic, and Differential Value of Enhanced MR Imaging in Guillain-Barré Syndrome}

Although we appreciate the excellent images of enhanced cranial nerves in pediatric patients with Guillain-Barré syndrome (GBS) and their contribution to the understanding of GBS as a continuum rather than distinct entities, we do not agree with Zuccoli et $\mathrm{al}^{1}$ that brain MR imaging should be considered in the routine evaluation in pediatric patients with GBS.

First, we would like to know why the authors performed enhanced MR imaging in the clinically diagnosed and laboratory-supported patients with GBS. GBS is a well-known inflammatory disease of peripheral nerves, including the spinal nerves and cranial nerves. Albuminocytologic dissociation is the hallmark of GBS. Moreover, electrophysiologic examinations may reveal demyelinating and/or axonal involvement. In the initial phase of GBS, breakdown of the bloodnerve barrier is the characteristic pathologic change, which may lead to enhancement of nerve roots. The study confirmed this. The diagnosis of GBS is based on clinical evaluation and electrophysiologic and CSF profiles.

Is there additional diagnostic value for GBS with an enhanced scan? In their series, $70.6 \%$ of the patients were found to have elevated CSF protein levels and $85.7 \%$ had positive electrophysiologic findings for GBS, which supported definite GBS. ${ }^{1}$ The authors did not provide MR imaging data of patients with negative CSF or electrophysiology findings. Asymptomatic enhancement of nerve roots was found in this study, and the enhancement had no clinical association. ${ }^{1}$ If no other features support the diagnosis of GBS, perhaps the asymptomatic enhancement may give a clue to the inflammatory process of GBS. Moreover, the authors did not provide the relationship between enhancement and the clinical and electrophysiologic features. Therefore, we cannot know if enhancement may help to determine the subtypes of GBS (acute inflammatory demyelinating polyneuropathy, acute motor axonal neuropathy, or acute motor-sensory axonal neuropathy).

Second, we wonder whether the enhancement may have prognostic value. As the authors noted, though prominent nerve root enhancement accompanied an increased severity of leg weakness, the correlation between intracranial findings and clinical outcomes was poorly established. However, they did not provide the details of the outcome of the patients with enhanced nerve roots. The prognostic value will be determined by adjusting confounding factors, such as baseline severity, electrophysiologic classification (demyelinating or axonal), baseline CSF protein levels, immune-modulating therapies, and the time between scanning and the initial symptoms.

Third, is the enhancement significant in differentiating GBS from chronic inflammatory demyelinating neuropathy (CIDP) presenting as GBS? A small portion of patients with CIDP may present with relatively rapid progression to the nadir within 4 weeks after the onset, but they will relapse or progress in the long run. ${ }^{2}$ Researchers have found several clues to differentiate it from GBS. ${ }^{3}$ Because enhancement and thickening of nerve roots is also seen in CIDP, ${ }^{4}$ the enhanced scan may help the differentiation. The grade of enhancement (absent, present, or strongly present) used in the Zuccoli study along with the morphology (thickening or not) may give a clue. Again, the time of scanning is important. We hypothesize that if there is a difference between GBS and CIDP presenting as GBS, the differential value will be maximal when scanning is performed in the "recovery" phase (eg, 6-8 weeks after the onset), when acute inflammation subsides in GBS while chronic inflammation persists in CIDP.

In conclusion, enhanced MR imaging need not be considered in the routine evaluation in GBS until its diagnostic, prognostic, and differential value is confirmed by prospective studies.

\section{References}

1. Zuccoli G, Panigrahy A, Bailey A, et al. Redefining the Guillain-Barré spectrum in children: neuroimaging findings of cranial nerve involvement. AJNR Am J Neuroradiol 2011;32:639-42

2. Odaka M, Yuki N, Hirata K. Patients with chronic inflammatory demyelinating polyneuropathy initially diagnosed as Guillain-Barré syndrome. J Neurol 2003;250:913-16

3. Dionne A, Nicolle MW, Hahn AF. Clinical and electrophysiological parameters distinguishing acute-onset chronic inflammatory demyelinating polyneuropathy from acute inflammatory demyelinating polyneuropathy. Muscle Nerve 2010;41:202-07

4. Duggins AJ, McLeod JG, Pollard JD, et al. Spinal root and plexus hypertrophy in chronic inflammatory demyelinating polyneuropathy. Brain 1999;122: 1383-90

$$
\text { H.-f. Li }
$$

Department of Neurology

X.-j. Ji

Department of Emergency Neurology Affiliated Hospital of Medical College Qingdao University, Qingdao Shandong, China 